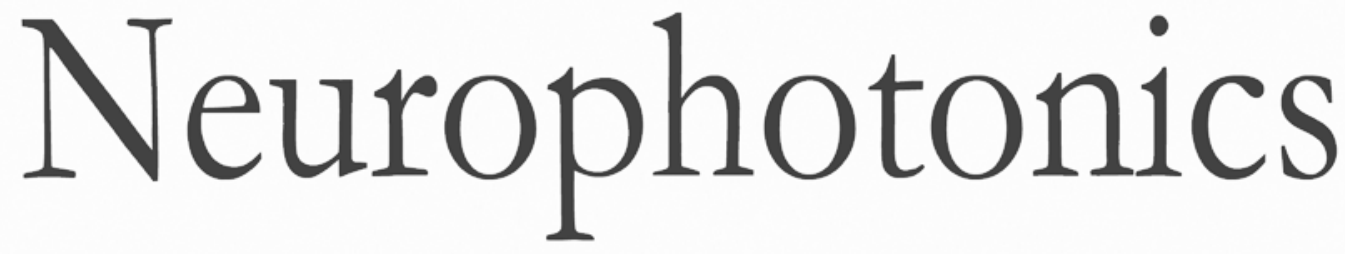

\title{
Open-source, cost-effective system for low-light in vivo fiber photometry
}

\author{
Kathryn Simone \\ Tamás Füzesi \\ David Rosenegger \\ Jaideep Bains \\ Kartikeya Murari
}




\title{
Open-source, cost-effective system for low-light in vivo fiber photometry
}

\author{
Kathryn Simone, ${ }^{a, \star}$ Tamás Füzesi, ${ }^{b, c}$ David Rosenegger, ${ }^{b, c}$ Jaideep Bains, $^{b, c}$ and Kartikeya Murari ${ }^{a, b, d}$ \\ aUniversity of Calgary, Biomedical Engineering Graduate Program, Calgary, Canada \\ bUniversity of Calgary, Hotchkiss Brain Institute, Calgary, Canada \\ 'University of Calgary, Department of Physiology and Pharmacology, Calgary, Canada \\ dUniversity of Calgary, Department of Electrical and Computer Engineering, Calgary, Canada
}

\begin{abstract}
Fiber photometry uses genetically encoded optical reporters to link specific cellular activity in stereotaxically targeted brain structures to specific behaviors. There are still a number of barriers that have hindered the widespread adoption of this approach. This includes cost, but also the high-levels of light required to excite the fluorophore, limiting commercial systems to the investigation of short-term transients in neuronal activity to avoid damage of tissue by light. Here, we present a cost-effective optoelectronic system for in vivo fiber photometry that achieves high-sensitivity to changes in fluorescence intensity, enabling detection of optical transients of a popular calcium reporter with excitation powers as low as $100 \mathrm{nW}$. By realizing a coherent detection scheme and by using a photomultiplier tube as a detector, the system demonstrates reliable study of in vivo neuronal activity, positioning it for future use in the experiments inquiring into learning and memory processes. The system was applied to study stress-evoked calcium transients in corticotropin-releasing hormone neurons in the mouse hypothalamus. @ 2018 Society of Photo-Optical Instrumentation Engineers (SPIE) [DOI: 10.1117/ 1.NPh.5.2.025006]
\end{abstract}

Keywords: optical fibers; biophotonics; fluorescence; optical signal detection; photometry; analog circuits.

Paper 17106RR received Jul. 14, 2017; accepted for publication Mar. 21, 2018; published online Apr. 12, 2018.

\section{Introduction}

In awake, freely moving animals, the neural recording modality of fiber photometry has become an important tool in identifying correlates of behavior. Fiber photometry has been used in mice to investigate sound-activation in the auditory cortex, ${ }^{1}$ transients in neural activity involved in the initiation of action, ${ }^{2}$ social interaction, ${ }^{3}$ and risk-taking. ${ }^{4}$

Engineering advances in the instrumentation include its integration with established methodologies such as electrophysiology ${ }^{5}$ to supplement the recording with a readout reflective of the "natural language" of neurons. Correction for extraneous transients caused by motion is achieved with multiple light sources and/or detectors. ${ }^{6}$ The ability to monitor several brain structures simultaneously with optical tools is achieved by implanting multiple fiber probes. Pashaie and Falk ${ }^{8}$ explored the concept of simultaneous control and monitoring by integrating optogenetic stimulation and fiber photometry in a single optical fiber probe.

These improvements enable neuroscientists to better identify, explain, and cause rapid transients in neural activity that correlate with behavior in real-time. However, there are comparatively fewer studies of slow changes in neural activity that engage in long-term and repeated photometric measurements across weeks and months. Such studies would deepen our understanding of the processes that underlie learning, memory, disease progression, and long-term or permanent changes in normal or pathological behavior. We approached the problem of adapting fiber photometry to long-term and repeated optical

*Address all correspondence to: Kathryn Simone, E-Mail: kathryn.simone@ ucalgary.ca measurements of neural activity by designing an optoelectronic system for fiber photometry that avoids exposing tissue to levels of light that are known to damage the optical reporter or the tissue. To avoid photobleaching in GFP-derived reporters such as GCaMP6s, excitation irradiances lower than $31.83 \mu \mathrm{W} \mathrm{mm}^{-2}$ are required $\left(1 \mu \mathrm{W}\right.$ for $200 \mu \mathrm{m}$-diameter probes). ${ }^{9}$ The level of input excitation light that causes phototoxicity has received less attention, but is assumed to occur at higher levels. ${ }^{10}$

The proposed design is based off of a custom lock-in amplifier (LIA) and was applied to experimentally investigate models of fluorescence detection in a scattering phantom, and in vivo, to investigate the correlates of stress. The design presented here marks a significant improvement over similarly specified designs ${ }^{11}$ in cost-efficacy and excitation power requirements, which will lower the barrier to widespread adoption of fiber photometry. The proposed system will henceforth be referred to as the cost-effective, in vivo, customizeable, low-light, fiber photometry system (CICLoPS).

This paper is organized as follows: Sect. 2 presents the design of the optoelectronic system and its subsequent characterization, and investigation of the collection profile of an optical fiber. Section 3 discusses the system's application to measuring calcium transients and its performance comparison to a commercial system.

\section{Methods and Results: Optoelectronic System Design and Characterization}

The photometry system design uses a modulated light-emitting diode (LED) as a light source, and a photomultiplier tube (PMT)

$2329-423 X / 2018 / \$ 25.00$ (C) 2018 SPIE 
is used as a detector. A custom LIA achieves coherent detection of optical signals. The LIA is implemented on a printed circuit board (PCB). These design choices drastically reduce the cost and complexity compared to ultrasensitive photometry systems proposed by others. ${ }^{11}$ The reporter for which this design is optimized is GCaMP6s, ${ }^{12}$ expressed in corticotropin-releasing hormone (CRH) neurons of the paraventricular nucleus of the hypothalamus (PVN) of a CRH-iresCre mouse. PVN CRH neurons are responsible for launching the endocrine response to stress and contribute to distinct behavioral repertoires after a stressful event. ${ }^{13}$

Full design documentation, assembly instructions, and a troubleshooting guide are available at a Github repository. ${ }^{14}$

\subsection{Optical System}

Figure 1(a) shows the delivery and collection paths for excitation and emission light in the fiber photometry system. To prepare the light source, the lens of an LED centered at $465 \mathrm{~nm}$ (LXML-PN02, Lumileds) was removed through gentle application of heat to expose the semiconductor die. The LED was soldered on a round board and mounted in a 1-in. lens tube. Coupling of the excitation light was achieved through collimation of the light with a lens (AC127-019-A, Thorlabs), employment of an excitation filter (470/40 nm, Chroma Technology), and a dichroic mirror (495LP, Chroma Technology) to direct the excitation light toward the coupling lens (F240FC-532, Thorlabs) for launching the excitation light into the optical fiber.

The modulated LED driver is realized using an operational amplifier (OPA171, Texas Instruments), the schematic for which is shown in Fig. 1(b), top. The noninverting terminal of the op amp is supplied with the modulation voltage waveform from a data acquisition card (USB-6361, National Instruments) running a custom $\mathrm{C}++$ continous output voltage routine for a $9-\mathrm{V}$ amplitude sine wave. This signal is copied to the inverting terminal and along with the $-12 \mathrm{~V}$ reference voltage causes a sinusoidal current through the resistor. This current is forced through the LED, generating near-sinusoidal optical output in phase with the modulating signal. Parameters of the modulation are controlled by changing the sine wave. Using a $39-\mathrm{k} \Omega$ resistor, the average current through the LED is $850 \mu \mathrm{A}$, which sets the average power at the output of the $200-\mu \mathrm{m}$ optical fiber to $100 \mathrm{nW}$. The stability of the light source was investigated through a 15-min continuous recording at the output of the optical fiber with a power meter (PM100, Thorlabs). Figure 1(b), bottom panel depicts the acquired, filtered trace. The drift in the excitation power over time as measured at the output of the fiber was found to be $1.12 \mu \mathrm{W} / \mathrm{W} / \mathrm{s}$.

Emission photons generated by the sample are collected by the optical fiber tip and guided back to the optical system for detection by a PMT (H10771-40P, Hamamatsu). These photons pass through the dichroic, and an emission filter $(515 / 30 \mathrm{~nm}$, Chroma Technology). The beam is then focused to lie within the photocathode with a third lens (AC254-040-A, Thorlabs). A 515/30 filter was used in place of the application of a wider $525 / 50$ emission filter $^{6}$ as it was found that spurious photons from standard fluorescent room lighting were strong at $543 \mathrm{~nm}$, and were ultimately being collected by the implanted optical probe. Though coherent detection attenuates these incoherent photons, they may introduce nonlinearities due to the limited dynamic range of the PMT. This effect is undesired and was eliminated with the alternate filter choice, but could also have been solved with a thicker application of dental cement used to secure the implant probe, alternate room lighting choices, or reduced PMT gain.

The optical design was realized with modular optomechanical components, shown in Fig. 1(c). A 0.48-NA, 200- $\mu$ m diameter optical fiber was selected. While larger diameter probes (400 $\mu \mathrm{m}$ in photometry and $600 \mu \mathrm{m}$ for deep-brain imaging applications) have been implanted in freely moving mice, we selected the small diameter for application in popular animal models such as mice, as $300 \mu \mathrm{m}$ is considered a conservative safe upper limit for small rodents. ${ }^{15}$

\subsection{Lock-in Amplifier and Electronics}

Coherent detection provides immunity to uncorrelated noise sources by modulating the excitation of an experiment at some carrier frequency, $f_{c}$, and then using a mixer and steep electronic filter to measure the amplitude of the resulting signal. Because white noise is, by definition, uncorrelated with any deterministic signal, employing such a detection scheme lowers the noise floor of a given system. In addition, modulating at $f_{c}$ shifts the signal away from DC and the $1 / f$ noise that is strongest at low frequencies. The result is increased sensitivity.

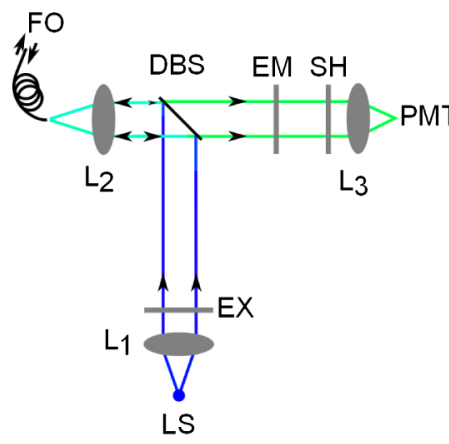

(a)

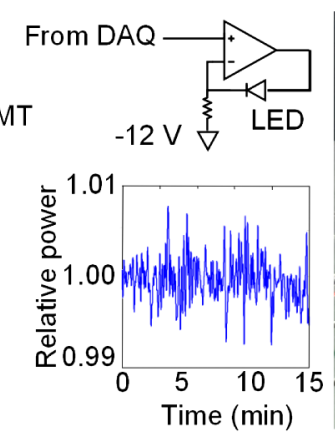

(b)

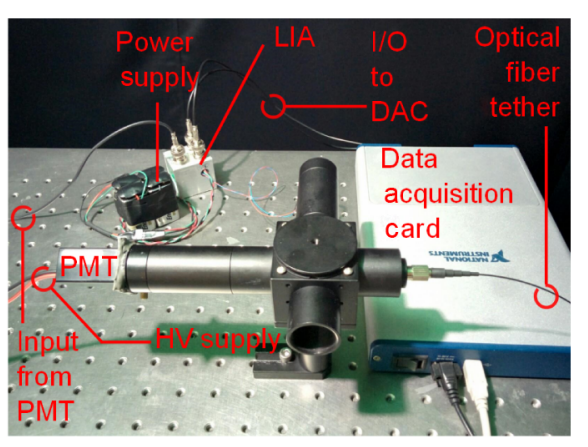

(c)

Fig. 1 Optical setup employed for the fiber photometry system. (a) Light path showing LS, LED light source; EX, excitation filter; DBS, dichroic beam splitter; FO, optical fiber tether; $\mathrm{SH}$, shutter; EM, emission filter; PMT, photomultiplier tube detector; and $\mathbf{L}_{1}, \mathbf{L}_{2}, \mathbf{L}_{3}$, collimating or focusing lenses. (b) (top) $L E D$ driver schematic for light source. (bottom) Light source power output recording during a 15-min period. Slow deviations from the mean excitation output power in the light source is $1.12 \mu \mathrm{W} / \mathrm{W} / \mathrm{s}$. (c) Photo of the optical system that realizes the specified light path with modular optomechanical components. 


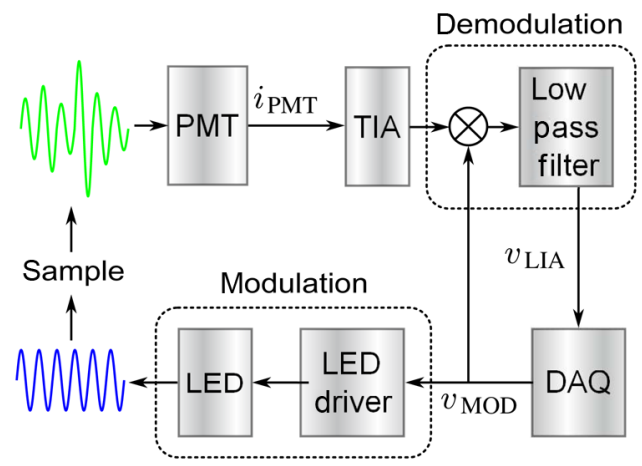

Fig. 2 Block diagram of proposed custom LIA to realize coherent detection scheme. DAQ, data acquisition card; PMT, photomultiplier tube; and TIA, transimpedance amplifier.

These "alternating current techniques," in the form of lock-in amplification, are therefore widely used in low-level optical detection applications ${ }^{16}$ including fiber photometry. ${ }^{3,8,11}$ Commercial LIAs are suitable for such applications, but are expensive and have additional functionality beyond what is required. We propose a cost-effective system that, as will be shown, also enables detection of calcium transients at light levels suitable to avoid photobleaching.

Figure 2 shows the schematic of the proposed design for monitoring low-level fluorescence in vivo. As explained in Sec. 2.1, a LED is an inexpensive light source that was chosen for this application because its power output is proportional to the current flowing through the device, enabling it to be easily modulated. The fluorescence generated coherently with the excitation of the tissue is detected by the PMT. The modulated photocurrent from the PMT is then transformed to a voltage signal by a transimpedance amplifier, in which the measured signal value is proportional to the feedback resistance. The noise in the transimpedance amplifier is dominated by Johnson noise, which increases with the square root of this resistance. The resultant SNR is therefore proportional to the square root of the transimpedance gain, according to

$\mathrm{SNR}=\frac{\left|I_{\mathrm{PMT}}\right| R_{f}}{\sqrt{4 k T B R_{f}}}$

where SNR is the signal-to-noise ratio of the transimpedance amplifier output, $I_{\mathrm{PMT}}$ is the input current signal, $R_{f}$ is the magnitude of transimpedance gain, $k$ is the Boltzmann constant, $T$ is the absolute temperature, and $B$ is the amplifier bandwidth. To maximize the SNR, the PMT current and the transimpedance gain should be as high as possible. However, since the coherent detection scheme used can not tolerate large distortions, the PMT must be used in its linear range, under $100 \mathrm{nA}$. With that constraint, $R_{f}$ and the amplifier's power supply were chosen to be $100 \mathrm{M} \Omega$ and $\pm 12 \mathrm{~V}$, respectively.

The operational amplifier (AD8065, Analog Devices) used in the front-end transimpedance amplifier was chosen for its low input bias current (1 pA), its ability to operate at the selected power supply, and for its wide gain-bandwidth product (145 MHz). Following analysis by Jung, ${ }^{17}$ the amplifier's phase margin was found to be $89 \mathrm{deg}$ at the unity loop gain frequency, well above the $45 \mathrm{deg}$ recommended for stability. The $f_{3 \mathrm{~dB}}$ bandwidth of the amplifier, that will inform the decision on modulation frequency, is then given as ${ }^{18}$

$$
f_{3 \mathrm{~dB}}=\sqrt{\frac{\mathrm{GBW}}{2 \pi R_{f}\left(C_{i}+C_{f}\right)}} \sqrt{1+\frac{1}{2 Q^{2}}\left(\sqrt{1-4 Q^{2}+8 Q^{4}}-1\right)},
$$

where quality factor $Q=\frac{\sqrt{\frac{\mathrm{GBW}}{f_{z}}}}{1+\frac{\mathrm{GBW}}{f_{p}}} . f_{3 \mathrm{~dB}}$ is predicted to be $5.32 \mathrm{kHz}$ with input capacitance $C_{i}=20 \mathrm{pF}$, feedback capacitance $C_{f}=0.3 \mathrm{pF}$, feedback resistance $R_{f}=100 \mathrm{M} \Omega$, input resistance $R_{i}=1 \mathrm{~T} \Omega$, and gain-bandwidth product GBW = $145 \mathrm{MHz} . f_{z}$ and $f_{p}$, the zero and pole frequencies of the noise-gain function, were found to be $7.84 \mathrm{~Hz}$ and $5.31 \mathrm{kHz}$, respectively.

A second-order, $5 \mathrm{kHz}$ Sallen-Key filter ${ }^{19}$ was realized to provide further band limitation of the incoming modulated signal. The total frequency response was then determined experimentally with an LIA (SR860, Stanford Research Systems). The TIA's measured cut-off frequency, $f_{3 \mathrm{~dB}}$, was $1.95 \mathrm{kHz}$, and the gain was found to be $160 \mathrm{~dB} \Omega$. The discrepancy in achieved bandwidth compared to theoretical bandwidth can be explained with larger parasitic capacitance $(0.8 \mathrm{pF})$ in the realized circuit than assumed during design $(0.3 \mathrm{pF})$.

Subsequent design choices were informed by the kinetics of the calcium reporter, GCaMP6s. Calcium transients reported by GCaMP6s are characterized by slow changes in fluorescence intensity. ${ }^{12}$ Treating this as a first-order system, the bandwidth (BW) of this signal was estimated from the reported rise times, $\tau_{\text {rise }}$, in the optical signal due to an action potential detected in mouse visual cortex in vivo ${ }^{12}$ using

$\mathrm{BW}=\frac{0.35}{\tau_{\text {rise }}}$.

This yielded a bandwidth of $1.95 \mathrm{~Hz}$. Optical transients measured from populations of active neurons would be slower, depending on the degree of synchronous activation.

In this topology, the same reference signal for driving the light source was used to perform demodulation. A modulation frequency of $211 \mathrm{~Hz}$ was selected for this purpose, to avoid powerline harmonics at multiples of $60 \mathrm{~Hz}$ (in North America). This modulation frequency is high enough to accommodate the calcium signal bandwidth. A multiplier integrated circuit (AD734, Analog Devices) performs the frequency mixing. A fourth-order, $10 \mathrm{~Hz}$ Sallen-Key Butterworth filter was designed to low pass filter the frequency-shifted signal, which entirely captures the calcium transient signals.

The modulation and demodulation circuits were implemented on the same PCB. $\pm 12 \mathrm{~V}$ was supplied by low-dropout regulators [MC7812 $(+12 \mathrm{~V})$ and MC7912 $(-12 \mathrm{~V}), \mathrm{ON}$ Semiconductor] each in turn supplied by two- $9 \mathrm{~V}$ batteries connected in series to avoid power line noise. The total input-referred noise in the 0.1 to $10 \mathrm{~Hz}$ band was measured to be $7.62 \mathrm{fA}$ (RMS). Using spacers, the PCB was mounted inside a $1.625^{\prime \prime} \times 1.5^{\prime \prime} \times 1.5^{\prime \prime}$ aluminum enclosure. BNC connecters were secured for the LIA inputs $\left[v(t)_{\mathrm{MOD}}\right.$ and $\left.i(t)_{\mathrm{PMT}}\right]$ and output $\left[v(t)_{\text {LIA }}\right]$. A custom $\mathrm{C}++$ program running a data acquisition card (USB-6361, National Instruments) supplies $v(t)_{\mathrm{MOD}}$ and saves samples of $v(t)_{\mathrm{LIA}}$ at 100 samples per second to. bin files. These files are read by custom MATLAB ${ }^{\circledR}$ scripts. 


\subsection{Collection Efficiency with Probe-Structure Separation}

Single-fiber photometry for neural recording involves implanting an optical fiber probe in tissue such that it can collect fluorescence emitted from genetically targeted populations. Stereotaxic coordinates of the mouse brain model, ${ }^{20}$ together with landmarks on the skull that serve as a reference, are used to predict the location of the structure in the brain for targeting of the probe. However, variability between animals cause the predicted coordinates to deviate from the actual coordinates, leading to improper placement of the probe. The challenge is compounded by the scattering of visible light by brain tissue: the optical fiber probe must be implanted very close to the population of cells to ensure delivery of excitation photons, and collection of emission photons from the structure of interest. Whereas, ideally, the probe tip would be placed as close as possible to the population of genetically targeted fluorescent cells, without crushing the cells of interest, it is much more likely that the probe tip is separated from the cells by some distance. This impedes the ability to detect and quantify transients in fluorescence intensity.

Figure 3(a) shows the ideal (perfect) and likely (imperfect) cases of probe placement resulting from implantation and illustrates how the ability to collect this fluorescence is reduced when the structure and the probe are separated. The natural loss of irradiance with distance from a source of finite size, as well as the scattering of light by tissue, reduces the number of photons that will be collected by the probe. Knowledge of the effect of probe parameters and placement on the ability to detect and quantify transients in fluorescence intensity would enable improved signal collection efficiency through application-specific probe design and surgical protocols. Moreover, a quantitative understanding of the ability to detect transients in fluorescence intensity from populations of genetically targeted cells in tissue will support subsequent histological assays to determine the cause of those transients in fluorescence.

The bidirectional process of delivering and collecting illumination from tissue with an optical fiber has been studied through the application of numerical methods for tissue oxygen saturation, ${ }^{21}$ and for fluorescence from single neurons with singlemode fibers. ${ }^{5}$ The collection volume of an optical fiber probe in tissues for detection of endogeneous fluorophores has been investigated both experimentally and through the development of mathematical models. ${ }^{22}$ Closed-form solutions for collection efficiency from fluorescent structures ${ }^{8}$ have been proposed; however, experimental validation is lacking.

We approached the problem of providing intuition for collection of fluorescence from arbitrary structures by developing and experimentally validating mathematical models. The analysis is adapted from a model of gradient-index, single mode fibers ${ }^{5}$ for quantifying the collection profile of a step-index, multimode fiber while taking into account scattering. Beginning with the ability to detect fluorescence from a fluorescent point source $Q(x, y, z)$ in front of the probe with a multimode fiber, the analysis then explores the collection of fluorescence from arbitrary spatial distributions of fluorescence by evaluating the appropriate integral.

Figure 3(b) shows the collection of fluorescence from a point source $Q(x, y, z)$ by a differential surface $d S$ located at point (a)
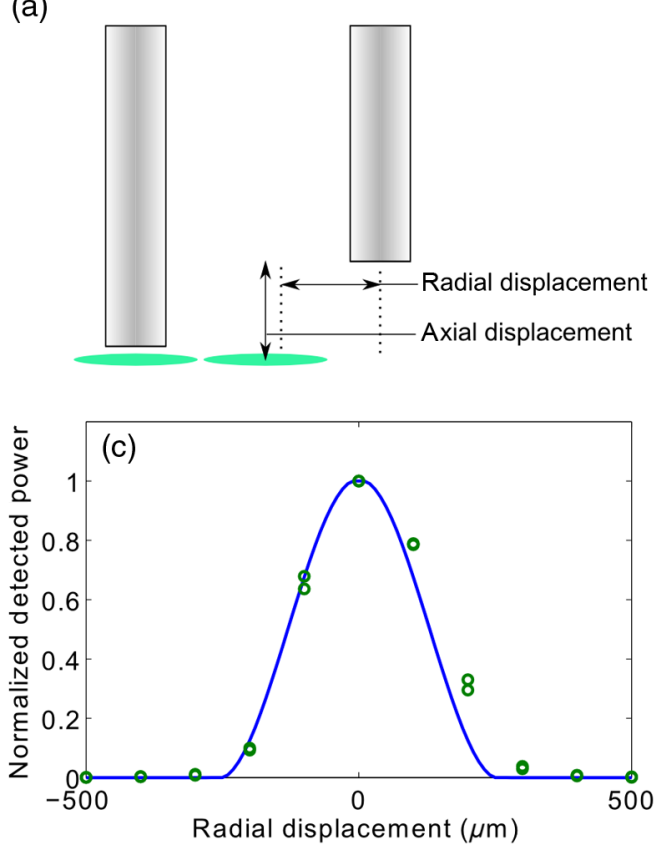

(b)
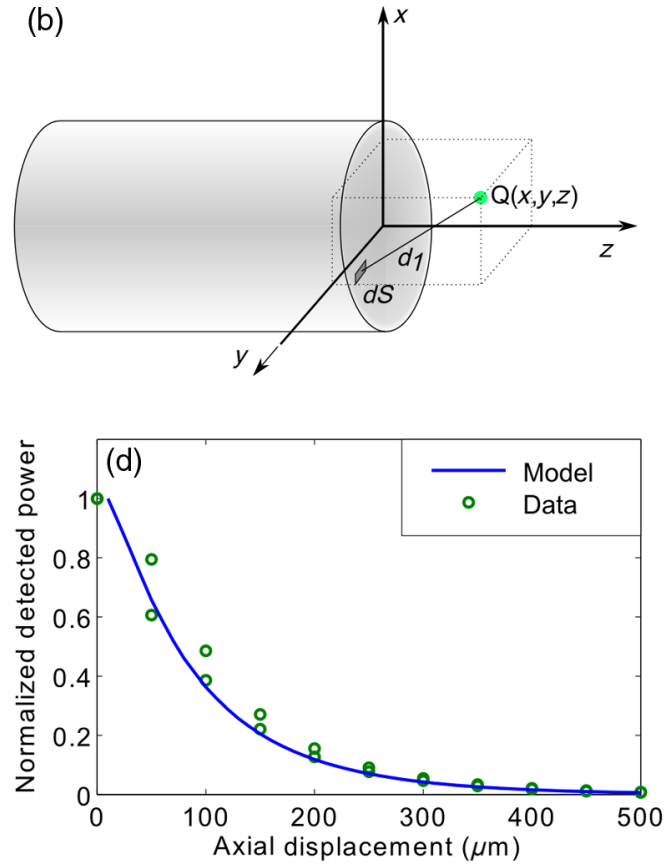

Fig. 3 (a) Fiber photometry involves implanting an optical probe near a spatially clustered population of genetically targeted fluorescent cells. (a) Left: Perfect implantation: ideally, the probe tip will be placed as close to the structure as possible without crushing the cells of interest. Right: Imperfect implantation: due to variability between animals, the actual coordinates of the population differ from those predicted using stereotaxic landmarks alone. The result is some combination of axial and transversal displacement that reduces the collection efficiency. (b) Collection of photons due to fluorescence from a point source $Q$ at $(x, y, z)$. The fluorescence is collected by the set of differential surfaces on the optical fiber tip $\mathrm{d} S=\mathrm{d} x \mathrm{~d} y$ separated from $Q$ by $d_{1}$. (c) Measured and modeled collection profiles from a fluorescent strip with radial displacement, with probe tip positioned at $z=50 \mu \mathrm{m}$. (d) Collection profile with axial displacement. 
$\left(x_{p}, y_{p}\right)$ on the tip of the optical fiber probe. The total power collected, $P_{c}$ depends on the local excitation intensity at that point, $I_{\mathrm{ex}}(x, y, z)$, and the emission coupling efficiency between the point source and the detector, $C_{m}(x, y, z)$

$P_{c}=\iint_{\text {fiber core }} I_{x}(x, y, z) C_{m}(x, y, z) \mathrm{d} x \mathrm{~d} y$.

The beam that emerges from the optical fiber was assumed to depend solely on the numerical aperture of the optical fiber and the refractive index of the medium in which the fiber was immersed. With intensity $I_{o}$ and waist $w_{o}$ at the tip of the probe (here assumed to be radius of the optical fiber core $r_{f}$ ), and radius $w(z)$ at distance $z$ from the tip for fiber with acceptance angle $\theta_{a}$, the intensity of excitation at a point $(x, y, z)$ in a scattering medium characterized by reduced scattering coefficient $\mu_{s}^{\prime}$ is given by $I_{x}(x, y, z)$

$I_{x}(x, y, z)=I_{o} \frac{w_{o}^{2}}{w(z)^{2}}\left\{1-\frac{1}{\left.1+e^{-\left[\sqrt{x^{2}+y^{2}}-w(z)\right.}\right]}\right\} e^{-\mu_{s}^{\prime} z}$,

where $w(z)=w_{o}+z \tan \theta_{a}$. The $\frac{w_{o}^{2}}{w(z)^{2}}$ term accounts for the reduction in local excitation intensity from initial intensity $I_{o}$ at $z=0$ due to the divergence of the beam with depth $z$, the second term approximates the spatial constraint of the excitation within the $x-y$ plane as a two-dimensional sigmoidal function, and $e^{-\mu_{s}^{\prime} z}$ approximates the transmission fraction due to scattering, as $z$ is assumed to be the distance traveled by most excitation photons.

The emission coupling fraction $C_{m}(x, y, z)$ approximates how many fluorescent photons are collected by the fiber. $C_{m}(x, y, z)$ is, in turn, determined by the fluorophore's quantum efficiency $\eta$ and absorption cross-section $A$, the radial distance $d_{1}$ separating the point and $d S$, the angle $\theta$ made by the line connecting $d S$ and $Q$ with the $z$ axis, specifically whether it lies within the acceptance cone defined by $\theta_{a}$, in turn determined by the fiber's numerical aperture (NA).

$C_{m}(x, y, z)=\frac{\eta A}{4 \pi d_{1}}\left[1-\frac{1}{1+e^{-\left(\theta-\theta_{a}\right)}}\right] e^{-\mu_{s}^{\prime} d_{1}}$,

where

$\theta=\tan ^{-1}\left[\frac{\sqrt{\left(x-x_{p}\right)^{2}+\left(y-y_{p}\right)^{2}}}{z}\right]$,

$d_{1}=\sqrt{\left(x-x_{p}\right)^{2}+\left(y-y_{p}\right)^{2}+z^{2}}$.

Integrating Eq. (4) over the fiber core then gives the total power collected by the probe due to a fluorescent point source.

We next computationally investigated the effect of transversal and axial displacement of optical probes near physiologically plausible fluorescent structures on the total collected optical power. We simulated the excitation and collection of fluorescence by the optical probe in front of neuronal populations, modeled as finite sheets of various dimensions, with numerical methods. We then experimentally investigated the dependence of axial and transversal displacement on the total power collected by an optical probe from a finite sheet of fluorescence in a scattering phantom. The numerical model was developed and tested for a $200 \mu \mathrm{m}$ optical fiber with $N A=0.48$, exciting fluorophores at $\lambda=475 \mathrm{~nm}$ with $P_{\mathrm{ex}}=15 \mathrm{nW}$ emerging from the tip of the probe. The scattering coefficient was assumed equal for both excitation and emission photons. As 2\% Intralipid was used as the scattering phantom for experimental work, the reduced scattering coefficient in the numerical model was set to $3.63 \mu \mathrm{m}^{-1} .^{23}$

To explore the physical accuracy of the numerical model, green masking tape (89097-930, VWR) that exhibits fluorescence when exposed to the system's light source was cut into either 1-mm-diameter disks or $250-\mu$ m-width strips and placed at the bottom of a petri dish with a microscope slide at its base. The strip width was selected to enable probing of axial resolution of a structure approximately as wide as the optical fiber, and the disk diameter was selected to approximate the shape of small fluorescent neural population such as the $\mathrm{CRH}$ neurons of the hypothalamus of the PVN. The petri dish was filled with the $2 \%$ Intralipid solution and in turn suspended in a larger container filled with the same intralipid solution. This "double immersion" was employed to simulate the situation in vivo, in which a fluorescence structure is suspended in a larger volume of scattering medium. The tip of a polished optical fiber probe was then positioned appropriately using a three-axis stage.

Figures 3(c) and 3(d) show the dependence of the collection profile on radial and axial positioning of the probe with respect to a linear and planar fluorescence source. As expected, the collection of the probe is maximized when the probe is centered on the structure in the $x-y$ plane (no radial displacement), and when axial separation is minimized. The collection efficiency is reduced to $50 \%$ when the probe is positioned with $\sim 125 \mu \mathrm{m}$ radial displacement, or with $75 \mu \mathrm{m}$ axial displacement.

\section{Methods and Results: In Vivo Application and Comparison with a Commercial Alternative}

The proposed optoelectronic system was applied to investigate the role of hypothalamic PVN CRH neurons in the stress response. These cells were targeted to selectively express the calcium-reporter GCaMP6s. Observed changes in fluorescence intensity due to calcium transients reflect underlying changes in neuronal firing activity of these cells. ${ }^{24}$

The CRH neurons of the PVN play an essential role in the neuroendocrine response to stress, which act on the order of minutes. ${ }^{25}$ However, recent studies have found evidence that these cells also play a role in mediating complex, rapid behavioral responses following exposure to stress. ${ }^{13}$

\subsection{Implantation Procedure}

All the experiments were approved by the University of Calgary Animal Care and Use Committee in accordance with Canadian Council on Animal Care guidelines. In a stereotaxic apparatus under isoflurane anesthesia, glass capillaries were lowered into the brain of 6- to 8-week old Crh-IRESCre; Ai14 mice [anteroposterior (AP), $-0.7 \mathrm{~mm}$; lateral (L), $-0.3 \mathrm{~mm}$ from the bregma; and dorsoventral (DV), $-4.5 \mathrm{~mm}$ from the dura]. Recombinant adeno-associated virus (AAV) carrying GCaMP6s (AAV9-CAG-GCaMP6s-WPRE-SV40; Penn Vector Core) or enhanced yellow fluorescent protein (EYFP) (pAAV-EF1adouble floxed EYFP-WPRE-HGHpA; Penn Vector Core) was pressure injected with Nanoject II apparatus (Drummond 
Scientific Company) in a total volume of $210 \mathrm{~nL}$. Animals were allowed to recover for 5 days and then handled for $\sim 5 \mathrm{~min}$ daily for four consecutive days before all behavioral testing.

\subsection{Photometric Data Acquisition and Analysis}

The optical fiber was prebleached by $5 \mathrm{~min}$ exposure to $2 \mathrm{~mW}$ optical power prior to the experiments. This prebleaching reduces the optical fiber's ability to photobleach further.

Raw photometric data were first processed with a $0.5-\mathrm{Hz}$ low-pass software filter and then transformed to changes in intensity as

$\Delta F / F=\frac{F-F_{o}}{F_{o}} \times 100 \%$,

where $F_{o}$ was computed from the mean intensity recorded during the first $100 \mathrm{~s}$ of the prebaseline recording.

Where applicable, changes in fluorescence intensity were further quantified using a measure of contrast-to-noise ratio, $\mathrm{CNR}$, defined as the ratio of the difference in mean signal values between two time periods, to the quadrature-averaged standard deviation of the traces from the two time periods.

$$
\begin{aligned}
& \mathrm{CNR}=\frac{\Delta F}{N}, \\
& =\frac{\mu_{2}-\mu_{1}}{\sqrt{\frac{1}{2}\left(\sigma_{1}^{2}+\sigma_{2}^{2}\right)}},
\end{aligned}
$$

where $\mu_{1}$ and $\sigma_{1}$ are the mean and standard deviation of the fluorescence signal during the first time period, and $\mu_{2}$ and $\sigma_{2}$ are the mean and standard deviation of the fluorescence signal during the second time period.

\subsection{In Vivo Recording}

Contextual fear conditioning occurs when an animal is placed in a new environment, exposed to an aversive stimulus, and then removed. Upon return to this context, the animal typically exhibits passive defensive behaviors (freezing). The animal remembers the last encounter with the environment and associates it with the bad experience. In other words, it has learned that the context is harmful. This paradigm is applicable here to help understand the role of $\mathrm{CRH}$ neurons in stress-mediated responses to conditioned fear. We delivered several footshocks (aversive stimulus) in a footshock cage (new environment). Upon reintroduction to the shock cage, the animal should exhibit changes in behavior due to memory of stress even in the absence of footshocks, and trigger the stress memory associated with increased CRH neuron activity and intracellular calcium concentration in those cells. This will be seen as increased fluorescence intensity in GCaMP6s animals, but no change in fluorescence detected in EYFP (control) animals.

The protocol used to investigate the neural correlates of conditioned stress is shown in Fig. 4(a). CRH-Cre mice transfected with either GCaMP6s (experimental) or EYFP (control) through viral injection, were implanted with fiberoptic cannula implants (MFC-200/230-0.48-5mm-MF2.5-FLT, Doric Lenses) on day zero, following the protocol developed by Cui et al. ${ }^{10} \mathrm{On}$ day 10 , the animal was subjected to a series of footshocks $(0.5 \mathrm{~mA} \text { for } 2 \mathrm{~s}, 10 \text { times in } 5 \mathrm{~min})^{13}$ delivered in the shockcage.
On day 11 , fiber photometry measurements were taken upon reintroduction to the shockcage without the shocks.

The photometry recording consisted of a 5-min baseline recording in the animal's home cage, a 6-min exposure to conditioned stress, and followed by a 5-min postrecording in the home cage again. For these experiments, the input excitation power, $P_{\mathrm{ex}}$, was $1 \mu \mathrm{W}$.

Figure 4(b) shows representative traces of fluorescence intensity when animals were reintroduced to the environment in which they had been subjected to a footshock stress. Reintroduction of the GCaMP6s animals to the context in which they had previously experienced a stress resulted in increased fluorescence intensity with a close time-correlation to the duration of the stress (GCaMP6s: $\mathrm{CNR}=7.27 \% \pm 1.47 \%, n=3$; EYFP: CNR $=-0.249 \%, n=1)$. These elevated levels of fluorescence were not seen in the control group. The trace obtained in the EYFP case does, however, exhibit large and rapid fluctuations in fluorescence intensity that may be due to artifacts of biological (such as hemodynamics) or nonbiological origin (movement and fiber bending).

\subsection{Performance Comparison}

We next sought to evaluate the system against other reported solutions. We applied it experimentally to a number of tasks alongside a popular commercially available alternative system. The alternative is based off of a Doric Lenses solution with a Tucker-Davison Technologies processor (RZ5), an appropriate system for comparison as it is also used in the application of recording GCaMP6s transients in vivo. The system used here was implemented as described in Supplementary Materials prepared by Lerner et al. ${ }^{6}$

We first investigated how performance compares between the two systems across their respective dynamic ranges, by recording over a range of excitation light conditions. A 3-nM solution of fluorescein was used as the source of fluorescence as this concentration was found to produce similar intensity to calciumsaturated GCaMP6s observed in vivo.

We then quantified how well the two systems can measure changes in fluorescence intensity in vivo using excitation powers that deliver similar SNR. To do this, we recorded the calcium activity in a CRH-iresCre GCaMP6s mouse upon transitioning to a new environment. As encountering a new environment increases arousal, CRH activity should increase for the duration of the encounter. We used both systems to capture separate segments of the recording, allowing a direct comparison of sensitivity between the two systems.

Finally, we applied both systems at their nominal excitation powers (CICLoPS, $100 \mathrm{nW}$; Doric, $30 \mu \mathrm{W}$ ) to the task of detecting neural correlates associated with innate stress from a nociceptive stimulus. This involved recording from animals while delivering a series of $102-\mathrm{s}, 0.5 \mathrm{~mA}$ footshocks.

Figure 4(c) shows the photon transfer curves, which plots noise versus signal on logarithmic axes, obtained from the two systems. The slope of this curve should be 0 in the read noise limited region and $1 / 2$ in the shot noise limited region. The data points for each system are annotated with the input excitation power in $\mu \mathrm{W}$ and resultant SNR in parentheses. The Doric-based solution exhibits read-noise limited behavior at lower excitation intensities but approaches shot-noise limited behavior at higher intensities. CICLoPS is nearly shot-noise limited over its entire dynamic range. The commercial system can achieve the high SNRs of CICLoPS but only at fairly high 

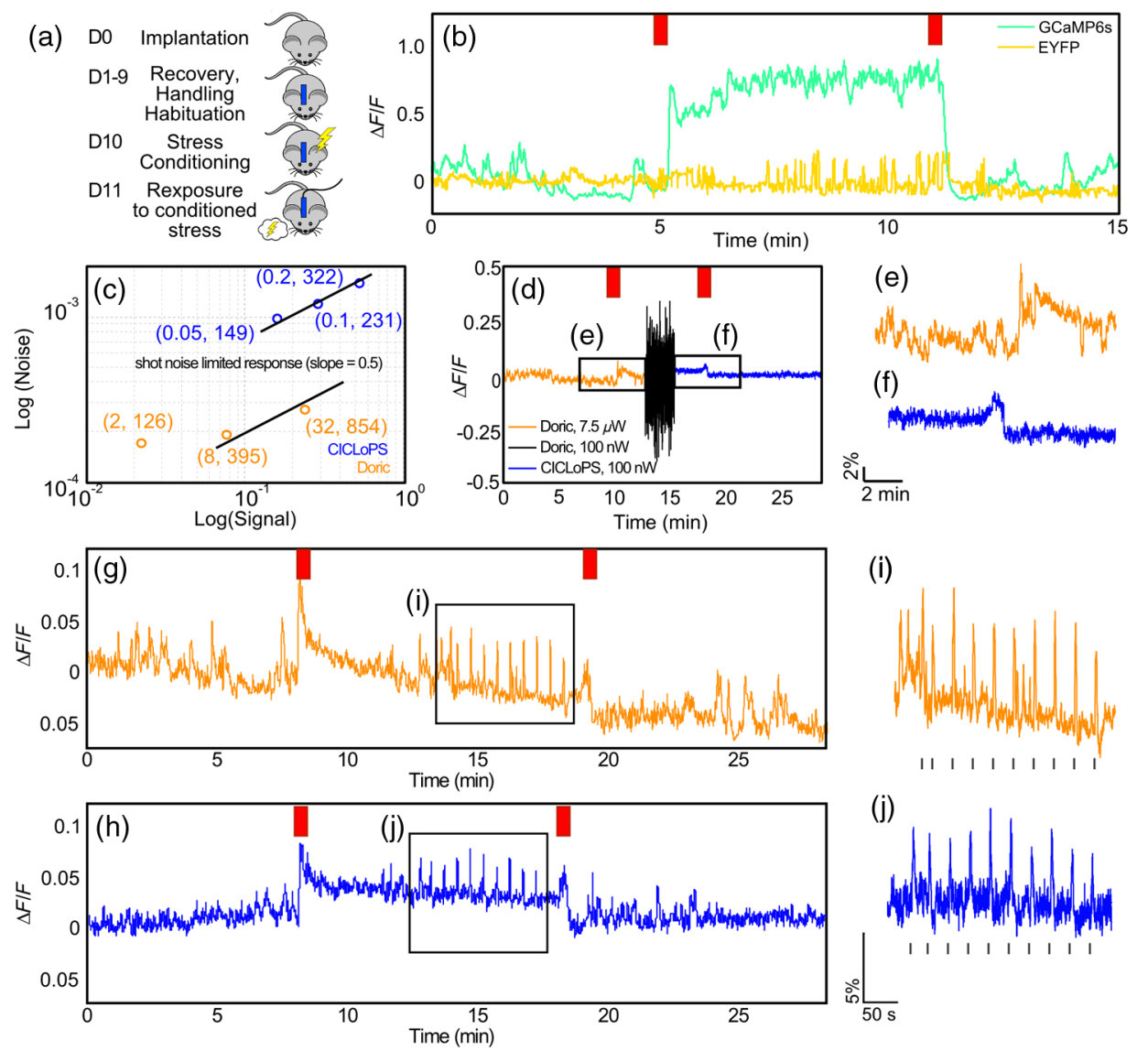

Fig. 4 Response of GCaMP6s-targeted PVN CRH cells in vivo and results from performance comparison with a commercial system. (a) Experimental protocol following viral injection of the GCaMP6s vector, for which (b) representative traces from a GCaMP6s (green) and EYFP (orange) CRH-Cre mouse. Red bars denote transfer to or from the footshock cage that provides the conditioned stimulus. (c) Photon transfer curves for the two systems compared in this study were generated over a range of excitation powers appropriate to each system. Numbers indicate (power in uW, SNR). (d) Recording from a single GCaMP6s mouse using both systems to capture different segments of a continuous experiment. (Red bars denote transfer to or from a new environment.) (e), (f) detailed traces at transition times. Recordings made by the Doric solution at $30 \mu \mathrm{W}$ (orange, g) and CICLoPS at $100 \mathrm{nW}$ (blue, h) during delivery of a series of footshocks. (i), (j) detailed traces during the footshock-delivery interval; black bars denote shock times (shock duration not to scale). CICLoPS offers similar performance as Doric at much lower excitation power leading to much lower photobleaching.

excitation powers. At $100 \mathrm{nW}$ excitation power, CICLoPS produced an SNR of 231.

Figure 4(d) shows the changes in fluorescence intensity recorded from a CRH-iresCre GCaMP6s mouse transitioned from its home cage to a new environment and then returned to its home cage, using both systems to capture different segments of the recording. For the CICLoPS system, $100 \mathrm{nW}$ was used, and for the Doric-based solution, 7.5 $\mu \mathrm{W}$. Recording first with the Doric solution operating at $7.5 \mu \mathrm{W}$, a baseline recording was taken from the animal in its home cage for $10 \mathrm{~min}$, then transitioned to a new environment at $t=10 \mathrm{~min}$. At $t=13 \mathrm{~min}$, the Doric system's excitation power was abruptly changed to $100 \mathrm{nW}$. At $t=15 \mathrm{~min}$, the optical tether connector was switched to CICLoPS operating at $100 \mathrm{nW}$. The traces obtained from CICLoPS and Doric operating at $7.5 \mu \mathrm{W}$ were transformed to changes in fluorescence intensity. No baseline measurement was obtained for the Doric solution at $100 \mathrm{nW}$. The trace depicted for the system operating at $100 \mathrm{nW}$ was transformed to $\Delta F / F$ to match the \% increase obtained for the system at $7.5 \mu \mathrm{W}$ just prior to switching to $100 \mathrm{nW}$.
A $4.1 \%$ increase was detected by the Doric solution at $t=10 \mathrm{~min}$. This increase in fluorescence intensity was followed by a gradual decay, suggestive of photobleaching. The segment acquired with the Doric system operating at $100 \mathrm{nW}$ did not cause photobleaching, but produced an SNR of just 6.3. As a result the Doric system was incapable of resolving the small increase in the fluorescence at $100 \mathrm{nW}$. The switch to CICLoPS with $100 \mathrm{nW}$ excitation at $t=15 \mathrm{~min}$ showed an increase in fluorescence over the baseline and no evidence of photobleaching was seen. Finally, a decrease similar to the initial increase $(3.7 \%)$ was seen at $t=18$ min when the animal was moved back to the home cage.

Figures 4(g) and 4(h) show changes in fluorescence intensity recorded with the commercial alternative and CICLoPS from a mouse transitioned from its home cage to a new environment (at $t=8 \mathrm{~min}$ ), subjected to a series of 10 footshocks at $\sim 30 \mathrm{~s}$ intervals in that new environment, and then returned to its home cage (at $t=\sim 18 \mathrm{~min}$ ). The systems are operated at nominal excitation powers $100 \mathrm{nW}$ for CICLoPS and $30 \mu \mathrm{W}$ for the Doric solution. 


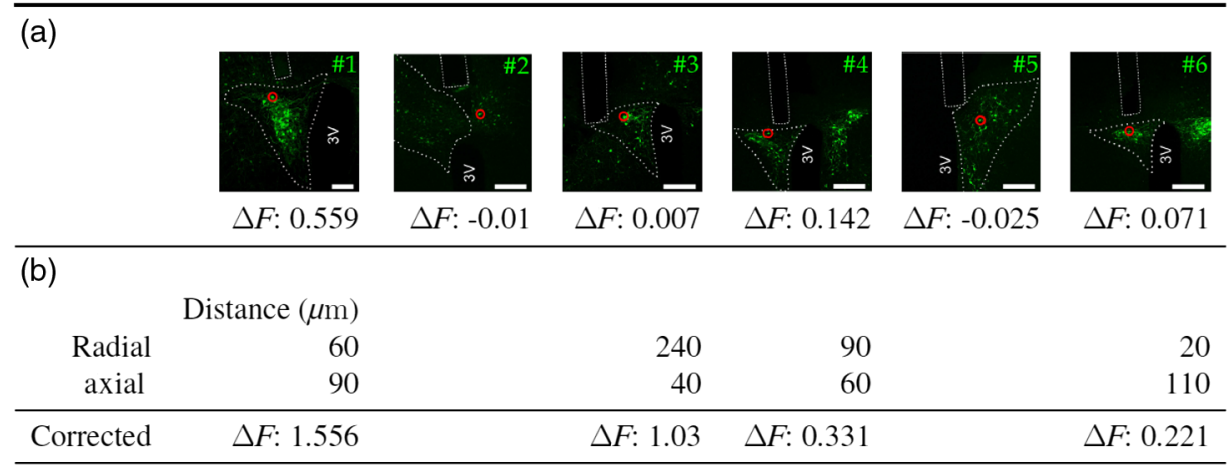

Fig. 5 (a) Identification of CRH neurons expressing GCaMP6s in the hypothalamic PVN of the hypothalamus of a mouse, and location of implanted fiber optical probe, 3V: third ventricle. The PVN is the cluster of fluorescent cells outlined in the micrograph for \# 1. Scale bars: \#1 $100 \mu \mathrm{m}$, \#2 to $6200 \mu \mathrm{m}$ (b) $\Delta F$ corrected for radial and axial probe misalignment using experimentally validated theoretical models of fluorescence detection in each animal.

Figures 4(i) and 4(j) show detailed traces during the shock delivery interval. Each footshock was followed by a rapid increase in fluorescence intensity of short duration, of amplitude $3.23 \% \pm 0.51 \%$ for CICLoPS and $5.23 \pm 0.68 \%$ for Doric. The recording made with the Doric system lost $7.51 \%$ of its intensity over the 28 -min recording, but only a very small change $(0.2 \%$ increase) was observed with CICLoPS system operating at $100 \mathrm{nW}$ (as calculated with the mean intensities during the first and last $100 \mathrm{~s}$ of each recording). Nominal excitation light of the commercial system induces significant photobleaching, but $100 \mathrm{nW}$ preserves signal fidelity.

While CICLoPS enables recording at excitation powers sufficient to avoid photobleaching, measurements with the CICLoPS configuration during the footshock stress were of lower responsivity than those obtained with the commercial alternative. As the experiments in the animal were conducted sequentially, it is possible that the neural response to nociceptive stimulus had fallen due to some habituation effect. The discrepancy may also be explained by the different emission filters employed between the two systems for this experiment: CICLoPS was configured with a 515/30-nm filter whereas the Doric solution employs a wider $525 / 50 \mathrm{~nm}$ filter. The autofluorescence of the optical fiber employed is stronger in the $515 / 30 \mathrm{~nm}$ band, ${ }^{26}$ reducing the sensitivity to GCaMP6s transients.

These results show that CICLoPS can detect changes in fluorescence intensity in vivo with $100 \mathrm{nW}$ of input excitation power. We have not seen any evidence of photobleaching at this light level. The Doric system could also be operated at this light level, but it is not clear what changes, if any, could be detected with the poor SNR produced (6.3). To improve the SNR, the excitation power has to be increased more than 10 -fold to a few $\mu \mathrm{W}$, which then leads to photobleaching. This effect is pronounced in Fig. $4(\mathrm{~g})$, where exposure to $30 \mu \mathrm{W}$ for 28 min resulted in a $7.51 \%$ reduction in fluorescence intensity.

\subsection{CNR Correction}

We next sought to investigate the ability of the theoretical models for fluorescence detection to correct for probe misplacement in vivo in photometry recordings. Figure $5(\mathrm{~b})$ provides the results of a post-hoc correction on $\Delta F$ given the probe misplacement, using data from the contextual fear conditioning experiments, as estimated to the nearest $10 \mu \mathrm{m}$ and the measured $\Delta F$. The analysis was performed only on those recordings that resulted in a positive $\Delta F$, as those recordings that produced a negative $\Delta \mathrm{F}$ are suggestive of dynamics in macroscopic tissue heterogeneities such as blood volume changes. The distance used to calculate the signal reduction due to axial and radial misplacement is the distance between the center of the probe as shown on the micrograph to the closest, bright object in the image. Bright objects were identified by thresholding a grayscale version of the original micrographs, and are annotated with a red circle in Fig. 5(a). The correction reduced the within-group variability for $\Delta F$, suggesting evidence that application of the models can help uncover the true $\Delta F$ for nonideally placed probes.

\section{Conclusion}

In this work, a highly sensitive optoelectronic system for in vivo fiber photometry, and its application in awake, freely moving mice to interrogate the behavioral correlates of the stress response was described. The system was designed to detect changes in fluorescence from GCaMP6s in applications requiring low excitation light levels. Validation experiments were performed to experimentally investigate the computational models of collection efficiency of fluorescence in a scattering medium, to investigate calcium transients of $\mathrm{CRH}$ cells in mice in response to a conditioned stress, and to compare its performance to a commercially available alternative.

In vivo data revealed a distinction between the optical transients produced in GCaMP6s and EYFP (control) groups, suggesting that $\mathrm{CRH}$ neurons are indeed active during the re-exposure to the conditioned stress. CICLoPS was found to perform similarly with the commercially available alternative in a variety of in vitro and in vivo experiments at much lower excitation powers, although a reduction in sensitivity was revealed during the innate stress test due to habituation or different emission filters in the systems. Meanwhile, recording with the CICLoPS system did not induce photobleaching, whereas application of the Doric system, and particularly when operating at its nominal $30 \mu \mathrm{W}$ did show a decreasing trend in fluorescence intensity over the acquisition period. The application of the theoretical models of collection profile in tissue to in vivo data also shows promising, yet limited, ability to correct for misplaced probes. We believe the major limitation of the model lies in the assumption of tissue homogeneity, whereas the recorded traces show a likely susceptibility to changes in tissue 
Table 1 Comparison of materials costs between the proposed system with alternative solutions in USD, to the nearest $\$ 100$. Where applicable, the cost of software is included.

\begin{tabular}{lccc} 
Subsystem & This work & Lerner et al. $^{6}$ & Doric Lenses system \\
\hline Optics & 2100 & 6000 & 4600 \\
Electronics & 200 & & 2100 \\
Acquisition & 1800 & 14,800 & 7500 \\
Photodetector & 2800 & 1400 & $2 \times 1400$ \\
Total & 6900 & 22,200 & 17,000 \\
\hline
\end{tabular}

absorptivity, suggestive of blood flow volume effects (a macroscopic tissue heterogeneity).

The system was designed to offer a highly sensitive, yet costeffective alternative to existing commercial solutions. Table 1 provides a breakdown of the materials costs of proposed photometry systems in USD, showing close to a $60 \%$ improvement in cost-efficacy to the next most cost-effective system. A major cost of the alternatives is that of the data acquisition systems, and modulated light sources that inflate the cost of the optics in both alternatives. These analogous subsystems in the proposed system were built using off-the-shelf parts at a much lower cost, allowing for more of the experimentalist's budget to be devoted to the photodetector, improving overall system sensitivity. Full design and assembly documentation meanwhile enables rapid implementation by nonengineers, providing savings on indirect costs related to the time spent building and troubleshooting the system.

Design for cost-efficacy and low-light operation alone neglected a number of key features that have become standard for robust acquisition of photometry measurements. A coherent detection scheme enables immunity only to uncorrelated signals, be they stochastic (such as white noise or $1 / f$ noise) or deterministic (for example, stray light). Movement artifacts and blood hemodynamics, in contrast, have a multiplicative effect on the detected baseband signal, and are therefore correlated with the desired signal. Correcting for these artifacts requires introducing reference signals to subtract their effect, such as the popular dual-excitation scheme for movement artifacts $^{6}$ or dual-detection schemes proposed for imaging modalities. ${ }^{27}$

Gross movement of the animal or bending of the fiber could be mitigated with a dual-excitation, isosbestic reference wavelength strategy proposed by Lerner et al. ${ }^{6}$ In this topology, a second LED at $405 \mathrm{~nm}$ excites endogenous fluorophores in tissue and is collected in the same emission band and detected by the PMT. A distinct modulation frequency for this excitation allows separation of the two signals by the detection electronics, and as $405 \mathrm{~nm}$ is an isosbestic point for GCaMP6s, the detected fluorescence intensity does not change with calcium saturation and is used as a reference signal to correct for movement artifacts. Due to the difference in tissue optical properties at the two wavelengths, particularly related to the absorption spectrum of hemoglobin, this strategy is limited to correction of artifacts of nonbiological origin.

On the other hand, photometric measurements may be obscured by dynamics in macroscopic tissue heterogeneities such as blood flow volume or refractive index due to the swelling of cells during action potential. This problem has been addressed for imaging modalities with a dual-detection scheme $^{27}$ to monitor fluorescence from coexpressed fluorophores, and could also be corrected by combining CICLoPS with wide-spectrum reflectance measurements and subsequent fitting of blood parameters. ${ }^{21}$

Finally, the responsivity of the CICLoPS configuration used for in vivo measurements in this work would likely be further improved by reconsidering the emission filter. The larger increases in fluorescence intensity detected by the commercial system in response to footshock is likely due to the use of a wider emission filter $(525 / 50 \mathrm{~nm})$ than employed in CICLoPS $(515 / 30 \mathrm{~nm})$ for these experiments. This filter was chosen to avoid a large peak at $543 \mathrm{~nm}$ typical of fluorescent room lighting systems, but also increases the sensitivity to autofluorescence from the optical fiber. Rather than using a filter that compromises sensitivity to the calcium reporter, thicker applications of dental cement with an added absorber could be used. Red food coloring was previously found to reduce transmission by $70 \% .^{26}$

To our knowledge, the instrument described here achieves photometric measurements under the lowest level light conditions reported, achieving superior SNR with excitation light at $100 \mathrm{nW}$ with a simple and cost-effective design achieved with a custom LIA on a PCB. This enables measurements to be taken without the nonlinearity induced by photobleaching, which simplifies subsequent data analysis and may enable the investigation of behavioral progressions that may take days or weeks to manifest. This work may help to extend fiber photometry to investigations that require long-term and repeated exposure of tissue to excitation light, such as those into learning and memory processes. The data collected from such studies would otherwise be inaccessible or difficult to interpret with the nonlinearities of photobleaching affecting day-to-day measurements. Cost-effective, low power photometry is needed to support techniques such as $24-\mathrm{h}$, in-cage monitoring that are increasing in popularity and require monitoring several animals simultaneously.

\section{Disclosures}

No conflicts of interest, financial or otherwise, are declared by the authors.

\section{Acknowledgments}

We thank Mio Tsutsui for performing the animal surgeries, Dinara Baimoukhametova for preparation of tissue slices, Richard Lushai and Linhui Yu for discussions on modeling, and Iain Barkley for helpful comments. The authors gratefully acknowledge funding by the Natural Sciences and Engineering Research Council (RGPIN/418976), the Canadian Microsystems Corporation emSYSCAN program, the Canadian Institutes for Health Research (CIHR 86501), and Brain Canada Neurophotonics Platform.

\section{References}

1. C. Grienberger et al., "Sound-evoked network calcium transients in mouse auditory cortex in vivo," J. Physiol. 590(4), 899-918 (2012).

2. G. Cui et al., "Concurrent activation of striatal direct and indirect pathways during action initiation," Nature 494(7436), 238-242 (2013).

3. L. A. Gunaydin et al., "Natural neural projection dynamics underlying social behavior," Cell 157(7), 1535-1551 (2014). 
4. K. A. Zalocusky et al., "Nucleus accumbens D2R cells signal prior outcomes and control risky decision-making," Nature 531(7596), 642-646 (2016).

5. Y. LeChasseur et al., "A microprobe for parallel optical and electrical recordings from single neurons in vivo," Nat. Methods 8(4), 319-325 (2011).

6. T. N. Lerner et al., "Intact-brain analyses reveal distinct information carried by SNC dopamine subcircuits," Cell 162(3), 635-647 (2015).

7. C. K. Kim et al., "Simultaneous fast measurement of circuit dynamics at multiple sites across the mammalian brain," Nat. Methods 13(4), 325-328 (2016).

8. R. Pashaie and R. Falk, "Single optical fiber probe for fluorescence detection and optogenetic stimulation," IEEE Trans. Biomed. Eng. 60(2), 268-280 (2013).

9. L. V. Doronina-Amitonova et al., "Fiber-optic probes for in vivo depthresolved neuron-activity mapping," J. Biophotonics 3(10-11), 660-669 (2010).

10. G. Cui et al., "Deep brain optical measurements of cell type-specific neural activity in behaving mice," Nat. Protoc. 9(6), 1213-1228 (2014).

11. L. Doronina-Amitonova et al., "Implantable fiber-optic interface for parallel multisite long-term optical dynamic brain interrogation in freely moving mice," Sci. Rep. 3, 3265 (2013).

12. T.-W. Chen et al., "Ultrasensitive fluorescent proteins for imaging neuronal activity," Nature 499(7458), 295-300 (2013).

13. T. Füzesi et al., "Hypothalamic CRH neurons orchestrate complex behaviours after stress," Nat. Commun. 7, 11937 (2016).

14. K. Simone, "CICLoPS photometry project," https://github.com/kpcsimone/CICLoPS (2018).

15. A. M. Aravanis et al., "An optical neural interface: in vivo control of rodent motor cortex with integrated fiberoptic and optogenetic technology," J. Neural Eng. 4(3), S143 (2007).

16. T. Vo-Dinh, Biomedical Photonics Handbook: Biomedical Diagnostics, CRC Press, Boca Raton, Florida (2014).

17. W. G. Jung, Op Amp Applications Handbook, Newnes, Burlington, Massachusetts (2005).

18. "Transimpedance noise calculation," http://www.jensign.com/noise/ index.html (19 February 2017).

19. R. P. Sallen and E. L. Key, "A practical method of designing RC active filters," IRE Trans. Circuit Theory 2(1), 74-85 (1955).

20. G. Paxinos and K. B. Franklin, The Mouse Brain in Stereotaxic Coordinates, Academic Press, San Diego, California (2004).

21. L. Yu et al., "In-vivo monitoring of tissue oxygen saturation in deep brain structures using a single fiber optical system," Biomed. Opt. Express 7(11), 4685-4694 (2016).

22. D. C. Tai et al., "Illumination and fluorescence collection volumes for fiber optic probes in tissue," J. Biomed. Opt. 12(3), 034033 (2007).
23. I. Driver et al., "The optical properties of aqueous suspensions of intralipid, a fat emulsion," Phys. Med. Biol. 34(12), 1927-1930 (1989).

24. H. Lütcke et al., "Inference of neuronal network spike dynamics and topology from calcium imaging data," Front. Neural Circuits 7, 201 (2013).

25. J. P. Herman and W. E. Cullinan, "Neurocircuitry of stress: central control of the hypothalamo pituitary adrenocortical axis," Trends Neurosci. 20(2), 78-84 (1997)

26. K. Ronayne, "Optoelectronic systems and applications for in vivo fiber photometry," Master's Thesis, University of Calgary (2016).

27. P. M. Goltstein et al., "In vivo two-photon ca2+ imaging reveals selective reward effects on stimulus-specific assemblies in mouse visual cortex," J. Neurosci. 33(28), 11540-11555 (2013).

Kathryn Simone is a PhD student in the biomedical engineering graduate program at the University of Calgary. She received her BSc degree in engineering from Memorial University of Newfoundland in 2014 and her MSc degree in biomedical engineering from the University of Calgary in 2017. Her current research interests include bioinstrumentation, neurophotonics, and computational neuroscience.

Tamás Füzesi is a neuroscientist at the Hotchkiss Brain Institute at the University of Calgary. He received his $\mathrm{PhD}$ in neuroscience from Semmelweiss University, Budapest, Hungary, in 2010. His current research interests include using optogenetic technologies to better understand stress circuits, plasticity, and behavior.

David Rosenegger is a research associate at the Hotchkiss Brain Institute at the University of Calgary. He received his PhD in neuroscience from the University of Calgary in 2009. During his postdoctoral research, he developed open-source two-photon microscopes and investigated astrocytic tonic regulation of brain blood supply. His current research interests include microscopy, optical instrumentation, and image processing.

Jaideep Bains, $\mathrm{PhD}$, is a professor of physiology and pharmacology at the University of Calgary. His group probes hypothalamic neurons from the level of synaptic mechanisms to awake, behaving animals with a particular interest in elucidating how neuromodulators released at the onset of stress alter subsequent stress responses.

Kartikeya Murari, PhD, is an associate professor of electrical and computer engineering at the University of Calgary. His lab develops innovative optical imaging and fiber instrumentation, as well as electrical recording and stimulation techniques for biomedical research applications. He is a member of IEEE and SPIE. 